\title{
Publisher Correction: Functional interaction between FUS and SMN underlies SMA-like splicing changes in wild-type hFUS mice
}

\author{
Alessia Mirra ${ }^{1,2}$, Simona Rossi ${ }^{3}$, Silvia Scaricamazza ${ }^{1,2}{ }^{2}$, Michela Di Salvio 4,5 , Illari Salvatori ${ }^{1}$, \\ Cristiana Valle ${ }^{1,6}$, Paola Rusmini ${ }^{7}$, Angelo Poletti ${ }^{7}{ }^{7}$, Gianluca Cestra ${ }^{4,5}$, Maria Teresa Carri ${ }^{1,2}$ \& \\ Mauro Cozzolino ${ }^{3}$
}

Correction to: Scientific Reports https://doi.org/10.1038/s41598-017-02195-0, published online 17 May 2017

The original version of this Article contained errors in the name of the author Mauro Cozzolino, which was incorrectly given as Maur o Cozzolino.

These errors have now been corrected in the PDF and HTML versions of the Article.

(i) Open Access This article is licensed under a Creative Commons Attribution 4.0 International License, which permits use, sharing, adaptation, distribution and reproduction in any medium or format, as long as you give appropriate credit to the original author(s) and the source, provide a link to the Creative Commons license, and indicate if changes were made. The images or other third party material in this article are included in the article's Creative Commons license, unless indicated otherwise in a credit line to the material. If material is not included in the article's Creative Commons license and your intended use is not permitted by statutory regulation or exceeds the permitted use, you will need to obtain permission directly from the copyright holder. To view a copy of this license, visit http://creativecommons.org/licenses/by/4.0/.

(C) The Author(s) 2018

\footnotetext{
${ }^{1}$ Fondazione Santa Lucia IRCCS, 00143, Rome, Italy. "Dipartimento di Biologia, Università di Roma "Tor Vergata", Rome, Italy. ${ }^{3}$ Istituto di Farmacologia Traslazionale (IFT), CNR, 00133, Rome, Italy. ${ }^{4}$ Istituto di Biologia e Patologia Molecolari (IBPM), CNR, 00185, Rome, Italy. ${ }^{5}$ Dipartimento di Biologia e Biotecnologia "Charles Darwin", Università di Roma "Sapienza", 00185, Rome, Italy. 'Istituto di Biologia Cellulare e Neurobiologia (IBCN), CNR, 00143, Rome, Italy. ${ }^{7}$ Dipartimento di Scienze Farmacologiche e Biomolecolari (DiSFeB), Centro di Eccellenza sulle Malattie Neurodegenerative, Università degli Studi di Milano, 20133, Milan, Italy. Maria Teresa Carrì and Mauro Cozzolino jointly supervised this work. Correspondence and requests for materials should be addressed to M.C. (email: mauro. cozzolino@ift.cnr.it)
} 